\title{
BLICKDIAGNOSE
}

Patient mit Adipositas permagna

\section{Woher kommen die auffälligen Hautveränderungen?}

Ein 49-jähriger Patient mit einem Bodymass-Index (BMI) von $65 \mathrm{~kg} / \mathrm{m}^{2}$ berichtete über auffällige Hautveränderungen im Bereich des Nackens (Abb. A) und der Achseln (Abb. B), die sich im Verlauf vieler Jahre entwickelt hatten. Inspektorisch zeigte sich eine derbe, hyperpigmentierte Haut an den genannten Stellen.

Bei dem Patienten bestand eine ausgeprägte Insulinresistenz. Aufgrund der Anamnese und dieser Befunde lässt sich hier die Diagnose einer Acanthosis nigricans benigna stellen.

Eine Acanthosis nigricans kann aber auch als paraneoplastisches Syndrom im Zusammenhang mit einer Vielzahl von
Tumoren auftreten. Eine solche sogenannte Acanthosis nigricans maligna entwickelt sich in der Regel recht kurzfristig.

\section{Keywords: Acanthosis nigricans}

- PD Dr. med. Andreas Barthel ${ }^{(1,2)}$, Prof. Dr. med. Stefan R. Bornstein $^{(2)}$, Prof. Dr. med. Georg Benker ${ }^{(1)}$

${ }^{(1)}$ Endokrinologikum Ruhr, Alter Markt 4, D-44866 Bochum ${ }^{(2)}$ Medizinische Klinik und Poliklinik III, Technische Universität Dresden, Universitätsklinikum Carl Gustav Carus, Fetscherstr. 74, D-01307 Dresden

\section{Ihr besonderer Fall?}

\section{Stellen Sie uns Ihren Fall vor}

Sicher sehen auch Sie ab und an einen besonders eindrucksvollen Befund in Ihrer Praxis. Fotografieren Sie ihn, schreiben Sie uns unter dem Stichwort Blickdiagnose, bei Veröffentlichung erhalten Sie einen Buchgutschein im Wert von 100 Euro.

MMW-Fortschritte der Medizin

E-Mail: Reinhold.Manhart@springer.com

Fax: 089/203043-31424 